\title{
How Does G-CSF Act on the Kidney during Acute Tubular Injury?
}

\author{
Masashi Nishida Kenji Hamaoka \\ Department of Pediatric Cardiology and Nephrology, Kyoto Prefectural University of Medicine Graduate School of \\ Medical Science, Kyoto, Japan
}

\section{Key Words}

Granulocyte colony-stimulating factor $\cdot$ Acute tubular injury $\cdot$ Acute renal failure $\cdot$ Bone marrow cell $\cdot$

Hematopoietic stem cell

\begin{abstract}
Recent findings in stem cell research have demonstrated multi-lineage plasticity of bone marrow cells, and also the contribution of hematopoietic bone marrow stem cells to the regeneration of injured organs including the kidney. These findings suggested the possibility of the use of granulocyte colony-stimulating factor (G-CSF) as a therapeutic option to regenerate injured organs. Recently, several studies regarding the effect of G-CSF on renal function have been reported in mouse models of acute renal failure. This series of experiments provided potentially important information regarding the treatment of patients with renal injury. This review summarizes the possible actions of G-CSF on the kidney, especially during acute tubular injury caused by toxic or ischemic insults.

Copyright $\odot 2006$ S. Karger AG, Base
\end{abstract}

\section{Introduction}

In the past, the discovery of granulocyte colony-stimulating factor (G-CSF) and its potential to regulate neutrophil production has opened an exciting new era for the management of patients with hematological and malignant diseases. Now, G-CSF is widely used clinically not only in neutropenic conditions due to chemotherapy but to mobilize hematopoietic stem cells (HSCs) from the bone marrow $(\mathrm{BM})$ to peripheral circulation for use in hematopoietic transplantation. Additionally, recent findings in stem cell research demonstrated multi-lineage plasticity of bone marrow cells (BMCs), and also the contribution of hematopoietic BM stem cells to the regeneration of injured organs including the kidney [1]. These findings suggest another clinical application of G-CSF as a therapeutic option to regenerate injured organs. In fact, the clinical utility of G-CSF for organ regeneration has vigorously been studied in myocardial infarction, though its effects are still controversial [2].

Recently, several studies regarding the effect of G-CSF on renal function have also been reported in mouse models of acute renal failure (ARF) [3-7]. The results of these studies, however, indicated contradictory effects of GCSF on renal function, i.e. G-CSF attenuates renal injury in some settings and worsens injury in another. Because G-CSF is often used clinically in conditions showing both renal injury and myelosuppression, as in cases with myelosuppressive nephrotoxic chemotherapy for malignancies, this series of experiments provides potentially important information regarding the treatment of patients with renal injury with or without myelosuppression. Concurrently, the question arises as to how G-CSF affects the kidney during acute tubular injury. In this ar-

\section{KARGER \\ Fax +41613061234 E-Mail karger@karger.ch} www.karger.com (c) 2006 S. Karger AG, Basel

$1660-2129 / 06 / 1044-0123 \$ 23.50 / 0$

Accessible online at:

www.karger.com/nee
Masashi Nishida, MD, $\mathrm{PhD}$

Department of Pediatric Cardiology and Nephrology

Kyoto Prefectural University of Medicine Graduate School of Medical Science

Kawaramachi-Hirokoji, Kamigyo-ku, Kyoto 602-8566 (Japan)

Tel. +81 75251 5832, Fax +81 75251 5833, E-Mail mnishida@koto.kpu-m.ac.jp 
Table 1. Effect of G-CSF on experimental ARF in mice

\begin{tabular}{|c|c|c|c|c|c|}
\hline & $\begin{array}{l}\text { Induction } \\
\text { of ARF }\end{array}$ & $\begin{array}{l}\text { G-CSF } \\
\text { treatment* } \\
\text { day }\end{array}$ & $\begin{array}{l}\text { Granulo- } \\
\text { cytosis }\end{array}$ & $\begin{array}{l}\text { Granulocyte } \\
\text { infiltration of } \\
\text { the kidney }\end{array}$ & $\begin{array}{l}\text { Effect of } \\
\text { G-CSF } \\
\text { on ARF }\end{array}$ \\
\hline Tögel et al. [3] & $\mathrm{I} / \mathrm{R}$ & $\begin{array}{r}-2 \text { to } 0 \\
0 \text { to } 2\end{array}$ & $\begin{array}{l}\text { present } \\
\text { absent }\end{array}$ & increased & $\begin{array}{l}\text { detrimental } \\
\text { no effect }\end{array}$ \\
\hline Nishida et al. [4] & cisplatin & 0 to 3 & absent & unchanged & alleviative \\
\hline Iwasaki et al. [5] & cisplatin & -5 to -1 & absent & - & alleviative \\
\hline Fang et al. [6] & folic acid & 0 to 4 & - & - & alleviative \\
\hline Stokman et al. [7] & $\mathrm{I} / \mathrm{R}$ & -5 to 3 & present & decreased & alleviative \\
\hline
\end{tabular}

$\mathrm{I} / \mathrm{R}=$ ischemia/reperfusion.

* The day of induction of ARF was day 0 . ticle, we review recent reports regarding the effects of GCSF on the kidney, and summarize the possible actions of G-CSF, especially during acute tubular injury caused by toxic or ischemic insults.

\section{Production of Endogenous G-CSF in the Kidney}

In normal humans, circulating levels of G-CSF are generally below $40 \mathrm{pg} / \mathrm{ml}$. However, under stress conditions, such as infection or following therapy with highdose cytotoxic agents, G-CSF levels increase dramatically and may exceed 2,000 pg/ml [8]. Cells of the monocyte/ macrophage lineage are among the most important sources of G-CSF, but they can also be produced by normal cells of mesodermal origin including vascular endothelial cells, fibroblasts, and mesothelial cells. Recent data by Zhang et al. [9] showed that renal ischemia-reperfusion injury in mice induces G-CSF gene expression by thick ascending limb cells in the kidney and increased peripheral serum concentration of G-CSF. Their data by in situ hybridization indicated that the increased G-CSF mRNA in the kidney was associated with renal tubules, although the possibility still exists that cell types other than renal origin such as macrophages, vascular endothelial cells, and fibroblasts may contribute to the production of G-CSF in the kidney in ischemia-reperfusion injury.

Recent reports, including studies on renal production of endogenous G-CSF, suggested that the in vivo response to acute renal injury involves not only the kidney but also extrarenal organs such as the liver, lung, spleen, brain, lymphoid tissues, and BM. These extrarenal responses to acute renal injury are stimulated by signals originating from the kidney and affect the course of ARF with con- comitant responses of the kidney itself. Thus, if G-CSF is produced by the kidney itself during acute renal ischemia-reperfusion injury, the possible action of this endogenous G-CSF is considered as one of the physiological responses of the kidney to the insult, affecting the course of renal injury.

\section{Effect of G-CSF Administration in Experimental ARF Models}

The effects of exogenous G-CSF administration on the course of ARF have been reported most recently in experimental mouse ARF models [3-7]. The results of these studies are summarized in table 1 . In all reports other than that by Tögel et al. [3], treatment with G-CSF was found to have a favorable effect on the course of ARF and resulted in decreased injury compared with control mice with the same insult to the kidney. In the report by Tögel et al. [3], however, the boosting of peripheral stem cell numbers by G-CSF was associated with increased severity of renal failure, and mortality, in ischemia-reperfusion injury in mice. On the other hand, in normal kidneys without insult, administration of G-CSF did not exert any detrimental effects [3]. Additionally, among reports that showed alleviative effects of G-CSF, there is still controversy regarding the mechanisms by which G-CSF exerts an alleviative effect on renal injury following insult. The contradictory results of these studies with experimental ARF models suggested complex effects of G-CSF on the kidney. The possible actions of G-CSF on the kidney during toxic or ischemic insults are discussed below. 


\section{Effect of Neutrophilia and Modulation of Neutrophil Function Induced by G-CSF}

G-CSF is believed to stimulate the BM to produce both neutrophils and macrophages. Furthermore, G-CSF appears to modulate certain neutrophil functions. Tögel et al. [3] deduced that the adverse effects of pharmacologic stem cell mobilization to renal function are mediated by the concomitant induction of marked granulocytosis, because identical ischemic injury in neutropenic mice resulted in milder renal injury. In two reports with cisplatin-induced ARF models, including our report $[4,5]$, granulocytosis was absent. This is mostly likely because cisplatin itself exerted a myelosuppressive effect. Both of these reports showed alleviated renal injury with G-CSF treatment. There have also been several previous reports indicating that inflammatory responses elicited by GCSF-induced neutrophilia exacerbate renal injury in experimental animals and humans. In studies with warm renal ischemia-reperfusion injury in rats [10] and in renal transplant patients [11], worsening of renal function with the use of G-CSF paralleled induced neutrophilia. Therefore, stimulation of neutrophil function as the source of reactive oxygen species as well as increased numbers of circulating neutrophils has been considered responsible for the worsening of renal function associated with the use of G-CSF.

In the study by Stokman et al. [7], despite increased numbers of neutrophils in the circulation, infiltration of granulocytes into the injured kidneys was significantly decreased by G-CSF treatment. They concluded that the underlying mechanism for the alleviative effect of G-CSF for renal injury is based on altered inflammatory kinetics after the initial ischemic insult. In their model, circulating granulocytes from G-CSF-treated mice showed significantly lower expression of L-selectin, whereas expression of CD11b was unaffected compared with controls. Furthermore, the concentrations of interleukin-1 $\beta$ (IL$1 \beta)$ and the keratinocyte-derived chemokine in kidney homogenates were also unaffected by G-CSF treatment. Thus, they speculated that the lower degree of migration of granulocytes to the injured kidney is likely the result of decreased selectin-mediated rolling of granulocytes on the endothelium, and is not the result of suppression of inflammatory processes as a whole.

These reports by Tögel et al. [3] and Stokman et al. [7] suggested the detrimental role of granulocytes infiltrating into the kidney during renal ischemic injury. However, in another report by Tögel et al. [12], the upregulation of stromal cell-derived factor-1 (SDF-1) expression in the kidney, which can induce leukocytosis, has been shown after renal ischemic injury. In this study, because SDF-1 attracts cells such as HSCs and endothelial progenitor cells, and these cells may have renoprotective effects, they suggested that SDF-1 may be involved in the kidney repair in this model. As one of the explanations for this contradictory effect of SDF-1, they suggested the importance of the time frame in which SDF-1 responses occur. G-CSF can also induce mobilization of both granulocytes and BM stem cells to the kidney, and the effect of G-CSF on the kidney during acute renal injury may, at least in part, depend on the time frame of the G-CSF action.

\section{Effect of HSC Mobilization by G-CSF on Tubular Regeneration}

Previous studies showed the differentiation of BM-derived HSCs to renal tubular epithelial cells (RTECs) following renal injury [1], suggesting the possibility that the boosting of HSCs to peripheral circulation by G-CSF may accelerate tubular regeneration. However, this prospect is still controversial in light of the recent data which showed that HSCs did not differentiate to RTECs during repair of the postischemic kidneys $[13,14]$. In experimental mouse ARF models, Iwasaki et al. [5] and Fang et al. [6] reported that treatment with G-CSF significantly increased BM-derived RTECs and suggested the contribution of boosted circulating HSCs by G-CSF to the regeneration of injured tubules. In the report by Iwasaki et al. [5], treatment with G-CSF increased BM-derived RTECs in approximately $0.5 \%$ of total tubular cells in salinetreated mice to more than $4 \%$ in G-CSF-treated mice that had received a transplant of BMCs of enhanced green fluorescent protein (EGFP)-transgenic mice, and had ARF induction by cisplatin injection. Furthermore, co-treatment with macrophage colony-stimulating factor (MCSF) enhanced this effect of G-CSF. In the report by Fang et al. [6], the frequency of BM-derived RTECs increased from approximately $4.3 \%$ in controls to $5.0 \%$ in G-CSFtreated female mice that had been transplanted with male BMCs at 3 days after folic acid treatment. They also demonstrated that these BM-derived cells undergo DNA synthesis. Although these two reports showed BM-derived RTECs at frequencies more than the rates that are considered as the result of cell fusion, most of the RTECs were still resident renal cell-derived.

In the report by Stokman et al. [7], at 7 days after ischemic insult to the kidney, only a few BM-derived RTECs were observed in both control and G-CSF-treated female 
mice that received $\mathrm{BM}$ transplantation from male EGFPtransgenic mice. Despite significant enhancement of functional recovery of the kidney, the incorporation of RTECs from BM origin was not increased with G-CSF treatment in their study. They concluded that the underlying mechanism for the alleviative effect of G-CSF for renal injury is not based on increased HSC or other BM stem cell involvement but rather on altered inflammatory kinetics. Also in our study [4], although myelosuppressive effect by treatment with cisplatin was suggested from decreased peripheral blood leukocyte count, only a few HSCs infiltrated the kidney, even with G-CSF treatment, indicating that the effect of G-CSF is not due to increased HSC infiltration to the kidney. Thus, granted that the boosting of HSCs by G-CSF may possibly enhance tubular regeneration by BM-derived cells at least in some settings, the alleviative effect of G-CSF for renal injury seems not solely to be dependent on increased RTECs from BM origin, but rather on other mechanisms.

\section{Effect of G-CSF on Vascular Cells}

G-CSF stimulates proliferation and migration of microvascular endothelial cells [15]. This might facilitate renal inflammatory responses elicited by G-CSF. However, previous reports also showed that G-CSF stimulates angiogenesis in infarcted myocardium, cerebral ischemia and limb ischemia, which acts to promote functional recovery of the damaged tissue [16]. These observations are based on the fact that HSCs mobilized by G-CSF can also differentiate into vascular endothelial cells and vascular smooth muscle cells. In fact, recent reports showed, though to a small extent, the differentiation of HSCs to vascular endothelial cells in postischemic kidneys, and suggested the role of HSCs in vasculogenesis $[13,14]$. The action of G-CSF on vascular cells and its physiological role in damaged kidney is still unknown. Nevertheless, these reports indicate the need to consider the effects of G-CSF on renal vascular cells, which may ameliorate or exacerbate renal injury.

\section{Effect of G-CSF on Tubular Epithelial Cells}

A direct effect of G-CSF on RTECs has not been previously indicated, and whether RTECs express the G-CSF receptor has not yet been elucidated. However, the antiapoptotic effect of G-CSF has been reported on cardiomyocytes and neuronal cells. These anti-apoptotic ac- tions of G-CSF are considered to be closely related to the activation of Janus-kinase (Jak)-signal transducer and activator of transcription (STAT) signaling pathway through the G-CSF receptor [17]. In our study [4], mice treated with G-CSF had significantly lower numbers of apoptotic RTECs compared with control mice. This may suggest the anti-apoptotic effect of G-CSF to RTECs.

On the other hand, the possibility also exists that GCSF indirectly affects RTEC function via the production of other agents that directly act on RTECs. From this perspective, a recent report [18] showed the elevation of hepatocyte growth factor (HGF), a potent renotrophic factor with mitogenic and anti-apoptotic activities on RTECs, in the serum of normal donors for allogeneic peripheral blood stem cell transplantation during G-CSF administration. In our mouse model of ARF [4], however, the serum HGF level was significantly decreased in mice treated with G-CSF compared with control mice, suggesting that the alleviative effect of G-CSF to renal injury is not due to the increased production of HGF.

\section{Effect of G-CSF on T Cells}

In recent years, a growing body of experimental evidence unraveled a novel role of G-CSF in the regulation of $\mathrm{T}$ cell and dendritic cell function, and G-CSF is now being increasingly accepted as a regulator of immune responses. This G-CSF-mediated immune regulation includes the ability to alter the $\mathrm{T}$ cell cytokine secretion profile and promote differentiation of regulatory $\mathrm{T}$ cells and tolerogenic dendritic cells. Accordingly, previous reports also showed beneficial roles of G-CSF for the prevention and treatment of several immune-mediated diseases in animal models. The anti-inflammatory effect of G-CSF was also observed in a mouse model of lupus nephritis [19]. Although T cell-mediated mechanisms in acute renal injury are still largely unknown, a recent report by Liu et al. [20] showed a marked attenuation in cisplatin-induced acute renal injury in $\mathrm{T}$ cell-deficient mice compared with wild-type mice. Concomitant reductions of renal tumor necrosis factor- $\alpha$ (TNF- $\alpha$ ), IL$1 \beta$, and keratinocyte-derived chemokine protein expression were also observed in these mice, and renal injury was enhanced by adoptive transfer of $T$ cells to these mice. These data demonstrate that $\mathrm{T}$ cells are direct mediators of experimental cisplatin nephrotoxicity. Thus, G-CSF may also exert an anti-inflammatory effect through this immunoregulatory mechanism by $\mathrm{T}$ cells, and may act to attenuate renal injury. 
Fig. 1. Effect of G-CSF on the kidney following renal insult. Possible mechanisms of action of endogenous or exogenous G-CSF to the kidney after acute toxic or ischemic insult are depicted schematically. Solid arrows indicate the actions of G-CSF that have been shown in experimental models, and dotted arrows indicate the possible actions of G-CSF to the kidney that have been speculated based on previous reports. $\mathrm{BM}=$ Bone marrow; HSCs = hematopoietic stem cells; ROS = reactive oxygen species; RTCs = renal tubular cells; HGF = hepatocyte growth factor; $\mathrm{G}$-CSF $=$ granulocyte colony-stimulating factor. $\uparrow$ denotes increase and $\downarrow$ denotes decrease of each factor. See main text for further details.

\section{Conclusion}

Figure 1 summarizes the actions of G-CSF on the kidney during acute tubular injury that had been reported previously in experimental models, and also the possible mechanisms by which G-CSF may act on the kidney during acute injury. As is shown in figure 1, G-CSF may be a two-edged sword after renal injury: it exerts alleviative effects, and concurrently, exerts detrimental effects on the kidney. These data show that careful observation for renal function is necessary when we use G-CSF in patients with renal injury. We cannot directly extrapolate from these experimental data to human clinical settings; nevertheless, these give us considerable suggestions in treating patients with renal injury. In cases with ischemic renal injury, because the use of G-CSF usually induces neutrophilia and neutrophils elicit inflammatory responses that result in further injury both in experimental models and in humans $[3,10,11]$, the use of G-CSF should

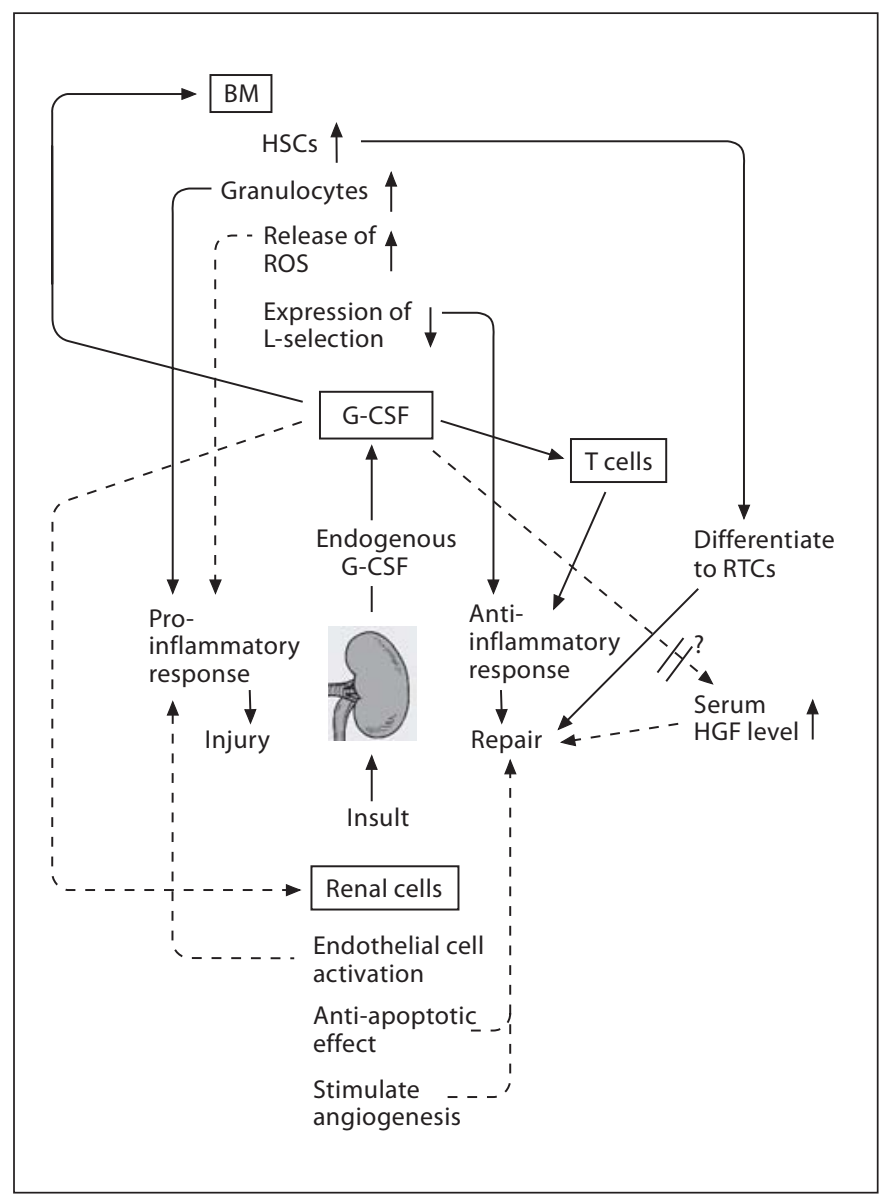

be avoided. In contrast, in cases with myelosuppressive conditions, e.g. with renal injury induced by myelosuppressive nephrotoxic chemotherapy, as long as its use is limited so as not to induce significant granulocytosis, the use of G-CSF may be approvable with close observations on renal function. Furthermore, G-CSF can be expected to attenuate renal injury in such conditions. Further studies regarding the effect of G-CSF on renal function in various clinical settings, including those with renal injury, will turn these potentially important experimental data to practical clinical utilization. 


\section{References}

-1 Lin F, Cordes K, Li L, Hood L, Couser WG, Shankland SJ, Igarashi P: Hematopoietic stem cells contribute to the regeneration of renal tubules after renal ischemia-reperfusion injury in mice. J Am Soc Nephrol 2003; 14:1188-1199.

-2 Zohlnhofer D, Ott I, Mehilli J, Schomig K, Michalk F, Ibrahim T, Meisetschlager G, von Wedel J, Bollwein H, Seyfarth M, Dirschinger J, Schmitt C, Schwaiger M, Kastrati A, Schomig A, REVIVAL-2 Investigators: Stem cell mobilization by granulocyte colonystimulating factor in patients with acute myocardial infarction: a randomized controlled trial. JAMA 2006;295:1003-1010.

>3 Tögel F, Isaac J, Westenfelder C: Hematopoietic stem cell mobilization-associated granulocytosis severely worsens acute renal failure. J Am Soc Nephrol 2004;15:1261-1267.

$\checkmark 4$ Nishida M, Fujimoto S, Toiyama K, Sato H, Hamaoka K: Effect of hematopoietic cytokines on renal function in cisplatin-induced ARF in mice. Biochem Biophys Res Commun 2004;324:341-347.

5 Iwasaki M, Adachi Y, Minamino K, Suzuki Y, Zhang Y, Okigaki M, Nakano K, Koike Y, Wang J, Mukaide H, Taketani S, Mori Y, Takahashi H, Iwasaka T, Ikehara S: Mobilization of bone marrow cells by G-CSF rescues mice from cisplatin-induced renal failure, and M-CSF enhances the effects of G-CSF. J Am Soc Nephrol 2005;16:658666.

-6 Fang TC, Alison MR, Cook HT, Jeffery R, Wright NA, Poulsom R: Proliferation of bone marrow-derived cells contributes to regeneration after folic acid-induced acute tubular injury. J Am Soc Nephrol 2005;16: 1723-1732.

7 Stokman G, Leemans JC, Claessen N, Weening JJ, Florquin S: Hematopoietic stem cell mobilization therapy accelerates recovery of renal function independent of stem cell contribution. J Am Soc Nephrol 2005;16:16841692.
-8 Kawakami M, Tsutsumi H, Kumakawa T, Abe H, Hirai M, Kurosawa S, Mori M, Fukushima M: Levels of serum granulocyte colony-stimulating factor in patients with infections. Blood 1990;76:1962-1964.

-9 Zhang Y, Woodward VK, Shelton JM, Richardson JA, Zhou XJ, Link D, Kielar ML, Jeyarajah DR, Lu CY: Ischemia-reperfusion induces G-CSF gene expression by renal medullary thick ascending limb cells in vivo and in vitro. Am J Physiol Renal Physiol 2004;286:F1193-F1201.

10 Tomikawa S, Akiyama N, Nagao T, Uchida $\mathrm{H}$ : Warm renal ischemia and reperfusion injury in rats treated with cyclophosphamide and/or granulocyte colony-stimulating factor. Transplant Proc 1993;25:3230-3233.

11 Minguez C, Mazuecos A, Ceballos M, Tejuca $\mathrm{F}$, Rivero M: Worsening of renal function in a renal transplant patient treated with granulocyte colony-stimulating factor. Nephrol Dial Transplant 1995;10:2166-2167.

12 Tögel F, Isaac J, Hu Z, Weiss K, Westenfelder C: Renal SDF-1 signals mobilization and homing of CXCR4-positive cells to the kidney after ischemic injury. Kidney Int 2005; 67:1772-1784.

13 Duffield JS, Park KM, Hsiao LL, Kelley VR, Scadden DT, Ichimura T, Bonventre JV: Restoration of tubular epithelial cells during repair of the postischemic kidney occurs independently of bone marrow-derived stem cells. J Clin Invest 2005;115:1743-1755.

14 Dekel B, Shezen E, Even-Tov-Friedman S, Katchman H, Margalit R, Nagler A, Reisner Y: Transplantation of human CD34+CD133+ hematopoietic stem cells into ischemic and growing kidneys suggests role in vasculogenesis but not tubulogenesis. Stem Cells DOI: 10.1634/stemcells.2005-0265.
- 15 Bocchietto E, Guglielmetti A, Silvagno F, Taraboletti G, Pescarmona GP, Mantovani A, Bussolino F: Proliferative and migratory responses of murine microvascular endothelial cells to granulocyte colony-stimulating factor. J Cell Physiol 1993;155:89-95.

- 16 Ohki Y, Heissig B, Sato Y, Akiyama H, Zhu Z, Hicklin DJ, Shimada K, Ogawa H, Daida H, Hattori K, Ohsaka A: Granulocyte colony-stimulating factor promotes neovascularization by releasing vascular endothelial growth factor from neutrophils. FASEB J 2005; 19:2005-2007.

17 Harada M, Qin Y, Takano H, Minamino T, Zou Y, Toko H, Ohtsuka M, Matsuura K, Sano M, Nishi J, Iwanaga K, Akazawa H, Kunieda T, Zhu W, Hasegawa H, Kunisada K, Nagai T, Nakaya H, Yamauchi-Takihara $\mathrm{K}$, Komuro I: G-CSF prevents cardiac remodeling after myocardial infarction by activating the Jak-Stat pathway in cardiomyocytes. Nat Med 2005;11:305-311.

- 18 Fujii K, Ishimaru F, Kozuka T, Matsuo K, Nakase K, Kataoka I, Tabayashi T, Shinagawa K, Ikeda K, Harada M, Tanimoto M: Elevation of serum hepatocyte growth factor during granulocyte colony-stimulating factor-induced peripheral blood stem cell mobilization. Br J Haematol 2004;124:190194.

19 Zavala F, Masson A, Hadaya K, Ezine S, Schneider E, Babin O, Bach JF: Granulocyte colony-stimulating factor treatment of lupus autoimmune disease in MRL-lpr/lpr mice. J Immunol 1999;163:5125-5132.

-20 Liu M, Chien CC, Burne-Taney M, Molls RR, Racusen LC, Colvin RB, Rabb H: A pathophysiologic role for $\mathrm{T}$ lymphocytes in murine acute cisplatin nephrotoxicity. J Am Soc Nephrol 2006;17:765-774. 